\title{
DEMO-Relevant Gyrotron Research at KIT
}

\author{
K. A. Avramidis ${ }^{1}$, G. Aiello ${ }^{1}$, P. T. Brücker ${ }^{1}$, B. Ell ${ }^{1}$, T. Franke ${ }^{2,3}$, G. Gantenbein ${ }^{1}$, G. Grossetti ${ }^{1}$, \\ S. Illy ${ }^{1}$, Z. C. Ioannidis ${ }^{1}$, J. Jin ${ }^{1}$, P. C. Kalaria ${ }^{1}$, A. Marek ${ }^{1}$, I. Gr. Pagonakis ${ }^{1}$, S. Ruess ${ }^{1}$, T. Ruess ${ }^{1}$,

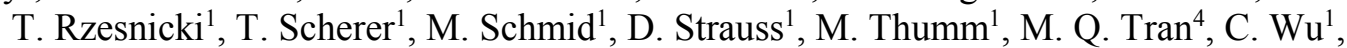 \\ and J. Jelonnek ${ }^{1}$ \\ ${ }^{1}$ Karlsruhe Institute of Technology, Karlsruhe, 76131 Germany \\ ${ }^{2}$ EUROfusion Consortium, Garching, 85748 Germany \\ ${ }^{3}$ Max-Planck-Institut für Plasmaphysik, Garching, 85748 Germany \\ ${ }^{4}$ École Polytechnique Fédérale de Lausanne, Lausanne, CH-1015 Switzerland
}

\begin{abstract}
The DEMO-relevant gyrotron research at Karlsruhe Institute of Technology is driven by the European concept for a demonstration fusion reactor (EU DEMO). This paper reports on the recent results of the theoretical and experimental studies towards the development of gyrotrons fulfilling the DEMO needs.
\end{abstract}

\section{INTRODUCTION}

$\mathrm{E}$ lectron Cyclotron Resonance Heating and Current Drive (ECRH \& CD) is expected to play a key role in the envisaged demonstration fusion power plants (DEMO) around the world. The required microwave power is of the order of 50-100 MW at frequencies even above $200 \mathrm{GHz}$ [15]. The gyrotron research at Karlsruhe Institute of Technology (KIT) is contributing to the European effort towards DEMO [5] by developing the advanced technologies necessary to address the challenges that ECRH \& CD poses on the microwave source, i.e. the gyrotron. These challenges stem from the required high values for power, frequency, efficiency, and reliability. The research at KIT is performed within the Work Package Heating and Current Drive (WPHCD), coordinated by the Power Plant Physics and Technology Department of the EUROfusion consortium. The target is the development of a $2 \mathrm{MW}, 170 / 204 \mathrm{GHz}$ Continuous-Wave $(\mathrm{CW})$ dual-frequency gyrotron, with a high overall efficiency $(\geq 60 \%)$ and with the option for fast frequency tunability in steps of $2-3 \mathrm{GHz}$, covering a range of $\pm 10 \mathrm{GHz}$ around the central frequencies. Operation at $\sim 240 \mathrm{GHz}$ is also under consideration.

To reach the target, technologies beyond the present state-of the-art are needed and are being developed [6]. In particular, the advanced coaxial gyrotron concept has been chosen to meet the challenge of the high frequency combined with high unit-power, which is very beneficial in terms of the required number of tubes and the reduction of complexity of the ECRH installation. Improvements on gyrotron cavity cooling by using mini-channels are under investigation. An innovative Multi-Stage Depressed Collector (MDC), based on a concept exploiting the $\mathbf{E} \times \mathbf{B}$ drift [7], is pursued for reaching high overall gyrotron efficiency, an essential feature for any future power plant. To support plasma instability control by finetuning the power deposition location with the frequency, the gyrotron should be capable of frequency step-tunability at 2 MW power levels. Therefore, a large broadband Brewsterangle Chemical Vapor Deposition (CVD) diamond window, compatible with a $63.5 \mathrm{~mm}$ diameter waveguide, is under development. For such a window, a large CVD diamond disk of $180 \mathrm{~mm}$ diameter and $\sim 2 \mathrm{~mm}$ thickness of optical grade with low intrinsic stresses is required. This specification is beyond the present state-of-the-art and a new field for the diamond manufacturers. Indispensable to the development of the EU DEMO gyrotron is the new $10 \mathrm{MW} \mathrm{CW} \mathrm{DC} \mathrm{test-stand}$ FULGOR at KIT, equipped with a new $10.5 \mathrm{~T}$ superconducting magnet. The test-stand is expected to be in operation in 2020 [8].

\section{RESEARCH RESULTS AND OUTLOOK}

The longer-pulse $170 \mathrm{GHz}, 2 \mathrm{MW}$ coaxial-cavity gyrotron at KIT [9], equipped with an advanced triode Magnetron Injection Gun (MIG) with coated emitter edges to minimize the influence of manufacturing tolerances and edge effects on the electron beam quality, was successfully tested in $\sim 1 \mathrm{~ms}$ pulses [10]. The gun was specifically designed to be capable of operating as a triode with the modulation anode at ground potential. This was done because, at the moment, a dedicated pulsed power supply for the modulation anode is not available at KIT. With the anode at ground potential, a power close to 2 MW with a total efficiency slightly below $45 \%$ in depressed collector operation was achieved. Next, adopting a more flexible configuration by using a DC power supply to set the modulation anode at negative potential (up to $-7 \mathrm{kV}$ ), an output power in excess of $2 \mathrm{MW}$ at $42 \%$ total efficiency with $30 \mathrm{kV}$ collector depression was demonstrated. This is a very promising result considering that, due to time limitation, higher values of the depression voltage to achieve improved efficiency were not tried. Further optimization is planned during the next experimental campaign. Also, the specifications for a proper pulsed power supply for the

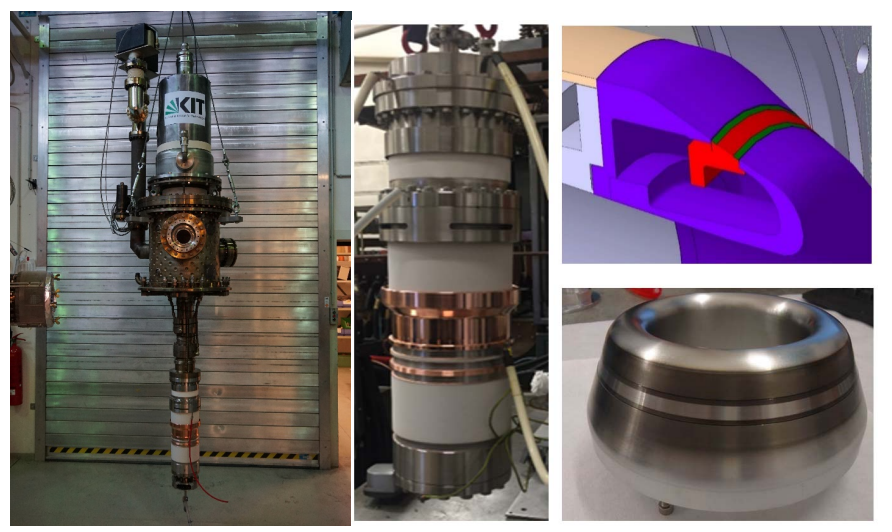

Fig. 1. The longer-pulse coaxial cavity gyrotron (left) equipped with the new coated triode gun (middle). Close-ups of the cathode design (right-top) and the cathode (right-bottom) are also shown. 
modulation anode are in preparation.

The extension of the pulse length up to $100 \mathrm{~ms}$ will also be pursued with the current configuration, as a first step towards the validation of the coaxial gyrotron technology in $\mathrm{CW}$ operation. In relation to this, and in view of further increase of the pulse-length in the future, theoretical studies on advanced cooling of the gyrotron cavity, based on mini-channels, resulted in a very promising design, planned to be tested using a cavity mock-up [11]. Also, multi-physics simulations of the cooling of the coaxial insert, addressing also the case of a misaligned insert, are ongoing [12].

In parallel to the experimental testing of the $170 \mathrm{GHz}$ coaxial gyrotron, the components for a $2 \mathrm{MW}, 170 / 204 \mathrm{GHz}$ dual frequency coaxial gyrotron were designed and numerically validated [13]. The operating parameters are DEMO-relevant and the design is based on the existing $170 \mathrm{GHz}, 2 \mathrm{MW}$ coaxial gyrotron, taking into account the magnetic field profile of the new $10.5 \mathrm{~T}$ superconducting magnet. The simulated performance of the design, as obtained by EURIDICE [14] (multi-mode beam-wave interaction simulations in the cavity and non-linear uptaper) with electron beam parameters calculated by ARIADNE [15], is shown in Table I. Apart from the aforementioned electron gun with coated emitter edges, the possibility to alternatively use an Inverse MIG (IMIG), manufactured at KIT [16], was also simulated. In principle, the IMIG offers the possibility for a larger emitter ring without increasing the overall gun size.

Using a newly upgraded mode generator set-up at KIT [17], the excitation of the selected very high-order mode for the $204 \mathrm{GHz}$ operation ( $\mathrm{TE}_{40,23}$ ) was possible. The set-up is now ready for low-power tests of the quasi-optical system of the dual-frequency gyrotron, once available.

A two-stage, short-pulse collector for the $170 \mathrm{GHz}, 2 \mathrm{MW}$ coaxial gyrotron, intended to be used for the forthcoming first experimental validation of the $\mathbf{E} \times \mathbf{B}$ MDC concept, is under development [18]. A first engineering design is shown in Fig. 2. With this configuration, a collector efficiency of $\sim 75 \%$, resulting in an overall gyrotron efficiency above $60 \%$, is expected. The various sub-components of the MDC are labelled in Fig. 2: the mirror box is grounded and insulated from the gyrotron body, which is at $+30 \mathrm{kV}$, while the first stage (d) and the vacuum envelope (c) of the MDC are electrically connected to the top of the mirror box (a). The lower part of the second stage (e) at $-12 \mathrm{kV}$ consists of bent metal plates, which create together with the first stage (d) the azimuthal E-field for the $\mathbf{E} \times \mathbf{B}$ drift, due to the helical spacing between them. The upper part of the second stage (h) is supported by the vacuum envelope by ceramic insulators $(\mathrm{g})$. A hollow coaxial insert (f) for the collection of reflected electrons is hung from the top of the vacuum envelop. The insert is also at ground potential.

Several CVD diamond growth experiments have been

Table I

Simulated performance of dual frequency 2 MW coaxial gyrotron design

\begin{tabular}{lllll}
\hline & \multicolumn{3}{c}{ Coated } & \multicolumn{2}{c}{ IMIG } \\
\hline Frequency [GHz] & 170.0 & 204.15 & 170.0 & 204.15 \\
Mode & $\mathrm{TE}_{34,19}$ & $\mathrm{TE}_{40,23}$ & $\mathrm{TE}_{34,19}$ & $\mathrm{TE}_{40,23}$ \\
RF cavity power [MW] & 2.60 & 2.08 & 2.57 & 2.08 \\
Interaction efficiency [\%] & 38.3 & 34.2 & 38.0 & 33.8 \\
\hline
\end{tabular}

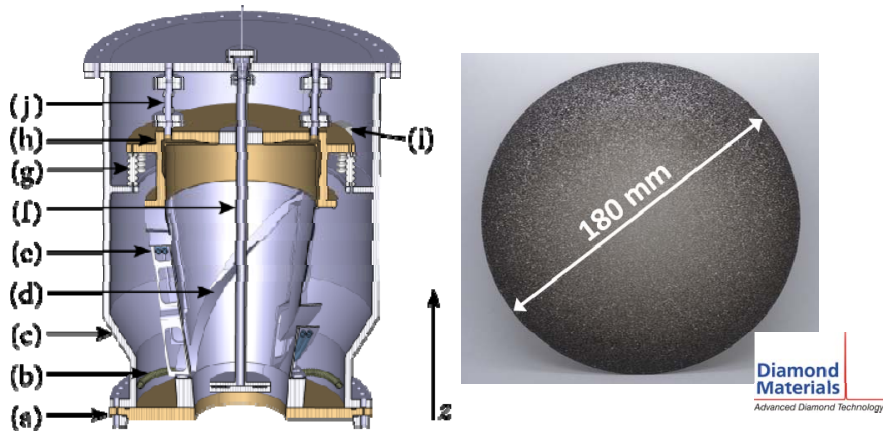

Fig. 2. Left: Engineering design proposal of a two-stage $\mathbf{E} \times \mathbf{B}$ collector. The components are explained in the text. Right: The world's first crack-free, free-standing CVD diamond disk of thermal quality, with $180 \mathrm{~mm}$ diameter and $\sim 2 \mathrm{~mm}$ average unpolished thickness.

carried out and are still ongoing by the industrial partner Diamond Materials GmbH, Freiburg, Germany, to optimize the parameters for diamond deposition on large $180 \mathrm{~mm}$ substrates [19]. The world's first crack-free diamond disk of thermal quality, with $180 \mathrm{~mm}$ diameter and $\sim 2 \mathrm{~mm}$ average unpolished thickness (Fig. 2), was produced in the microwave plasma reactor [20]. First experimental attempts to polish the $180 \mathrm{~mm}$ disks and loss-tangent measurements on polished disk extracts are underway, together with further optimization of the procedure for large diamond disk growth.

\section{ACKNOWLEDGMENT}

This work has been carried out within the framework of the EUROfusion Consortium and has received funding from the Euratom research and training programme 2014-2018 and 2019-2020 under grant agreement No 633053. The views and opinions expressed herein do not necessarily reflect those of the European Commission. Part of the simulations was performed on the EUROfusion High Performance Computer (Marconi-Fusion).

\section{REFERENCES}

[1]. Y. Wan et al., Nucl. Fusion, vol. 57, p. 102009, 2017.

[2]. K. Tobita et al., Fusion Sci. Tech., vol. 72, p. 537, 2017.

[3]. K. Kim et al., Nucl. Fusion, vol. 55, p. 053027, 2015.

[4]. EUROfusion, 2018, https://www.euro-fusion.org/eurofusion/roadmap/

[5]. G. Federici et al., Fus. Eng. Des., vol. 136, p. 729. 2018.

[6]. K. A. Avramidis et al., Nucl. Fusion, vol. 59, p. 066014, 2019.

[7]. I. Gr. Pagonakis et al., IEEE Trans. Plasma Sci. vol. 36, p. 469, 2008.

[8]. M. Schmid et al., Fusion Eng. Des. vol. 123, p. 485, 2017.

[9]. S. Ruess et al., Int. J. Microwave Wireless Techn., vol. 10, p. 547, 2018.

[10]. I. Gr. Pagonakis et al., this conference.

[11]. S. Illy et al., this conference.

[12]. P. C. Kalaria et al., "Multi-physics modelling of insert cooling system for a $170 \mathrm{GHz}, 2 \mathrm{MW}$ long-pulse coaxial-cavity gyrotron", IEEE Trans. Electron Devices, under review.

[13]. T. Ruess et al., 20 th Int. Vacuum Electronics Conf. (IVEC 2019), 28 April -1 May 2019, Busan, South Korea.

[14]. K. A. Avramides et al., EPJ Web of Conf. vol. 32, p. 04016, 2012.

[15]. I. Gr. Pagonakis and J. L. Vomvoridis, Joint $29^{\text {th }}$ Int. Conf. on Infrared and Millimeter Waves and 12 ${ }^{\text {th }}$ Int. Conf. on THz Electronics, 27 September-1 October 2004, Karlsruhe, Germany, proc. p. 657.

[16]. S. Ruess et al., IEEE Trans. Electron Devices, vol. 63, p. 2104, 2016.

[17]. T. Ruess et al., J. Infrared Millimeter THz Waves, vol. 40, p. 257, 2019.

[18]. C. Wu et al., Phys. Plasmas, vol. 26 p. 013108, 2019.

[19]. G. Aiello et al., "Diamond window technology for EC heating and current drive - state of the art", Fusion Sci. Techn., under review.

[20]. G. Aiello et al., "Towards large area CVD diamond disks for Brewsterangle windows", to appear at $14^{\text {th }}$ Int. Symposium on Fusion Nucl. Technology, 22 - 27 September 2019, Budapest, Hungary. 